\title{
PREVALENCIA DEL SINDROME DE TÚNEL CARPIANO Y SUS FACTORES ASOCIADOS EN ODONTÓLOGOS DE LA CIUDAD DE BUCARAMANGA Y SU ÁREA METROPOLITANA
}

${ }^{1}$ Andrea Rodríguez R., ${ }^{1}$ Katherine Morales P., ${ }^{1}$ Liseth P. Maldonado R., ${ }^{1}$ Gerardo E. Jiménez B., ${ }^{1}$ Luisa Fernanda Quiroz P., ${ }^{2}$ Sonia Constanza Concha $S .{ }^{3}$ Sandra Juliana Rueda V.

${ }^{1}$ Estudiante de $X$ semestre, F de Odontología, U. Santo Tomás, ${ }^{2}$ Odontóloga, U. Santo Tomás, Especialista en Educación y Comunicación para la Salud y el Bienestar, U. Industrial de Santander, Docente, U. Santo Tomás y U. Industrial de Santander, ${ }^{3}$ Odontóloga, U. Santo Tomás, Especialista en Patología Oral y Medios Diagnósticos, U. El Bosque, Docente U. Santo Tomás,

Autor responsable de correspondencia: Gerardo Jimenez B.

Correo electrónico: kike912@hotmail.com.

Premio a la mejor investigación en el Área de Seguridad Social y Servicios de Salud, categoría pregrado, en el XVI Encuentro de Investigación Odontológica ACFO, en Cartagena, septiembre de 2005

\section{RESUMEN}

Objetivo: Determinar la prevalencia del Síndrome del Túnel Carpiano (STC) y sus factores asociados en odontólogos de la ciudad de Bucaramanga y su área metropolitana.

Materiales y métodos: Se realizó un estudio observacional analítico de corte transversal. El universo se constituyó por 250 y la muestra por 156 seleccionados mediante muestreo aleatorio simple. Se analizó la relación de presencia de STC con cada variable, mediante test de chi2 y test exacto de Fischer. El análisis multivariado mediante un modelo de regresión binomial, considerado como variable de salida la presencia de STC y como variable explicatoria las relacionadas con factores de riesgo. Resultados: La prevalencia de STC en odontólogos de Bucaramanga fue de 15.8\%. El 40\% de los endodoncistas registraron STC; se observó que el 33\% de los odontólogos que registraban compromiso sistémico presentaban la condición de interés y que el $25 \%$ de las personas que usaban instrumental manual durante tiempos menores de $240 \mathrm{~min} /$ día registraban STC. Hubo una asociación seis veces mayor de tener STC en personas con compromiso sistémico y 0.13 de no tenerlo en personas que trabajan instrumental manual tiempos superiores a $240 \mathrm{~min} /$ día.

Conclusión: Se estableció que el compromiso sistémico fue un factor determinante en la aparición de STC y el uso de instrumental rotario por tiempos prolongados, fue un factor relacionado con el ejercicio profesional, asociado con el fenómeno. [Rodríguez A, Morales K, Maldonado LP, Jiménez GE, Quiroz LF, Concha SC, Rueda SJ. Prevalencia del sindrome de túnel carpiano y sus factores asociados en odontólogos de la ciudad de Bucaramanga y su área metropolitana. Ustasalud Odontología 2005; 4: 73 - 80]

Palabras clave: Síndrome del túnel carpiano, Dolor, Odontólogos, Riesgos profesionales.

\section{CARPAL TUNNEL SYNDROME PREVALENCE AND ITS ASSOCIATED FACTORS IN DENTISTS FROM THE CITY BUCARAMANGA AND IT'S METROPOLITAN AREA.}

\begin{abstract}
objective: To determine the prevalence of Carpal Tunnel Syndrome (CTS) and its associated factors in dentists of Bucaramanga and its metropolitan area.

Materials and methods: An analytical cross section observational study was made. The universe were 250 dentists and the sample 156 dentists that were chosen by a simple random sampling. The relationship between the presence of CTS with each variable was analyzed by means of test of chi2 and Fisher's exact test. The multivaried analysis included binomial regression model that considered as an exit variable the presence of CTS and like explicatory variables those related risk factors.

Results: The prevalence of CTS in dentists who work in Bucaramanga was $15.8 \%$. After analyzing the results according to each specialty, $40 \%$ of the endodontists had CTS, 30\% of the dentists who had systemic compromise had CTS and $25 \%$ of the dentists who used manual instruments during time periods less than $240 \mathrm{~min} /$ day had CTS. There was an association six times greater of having CTS in people with systemic compromise and 0.13 of not having CTS in persons who work with manual instruments during time periods greater than $240 \mathrm{~min} /$ day.

Conclusion: It was established that systemic compromise is determining factor in the appearance of CTS, the use of rotatory instruments for long time periods was a factor related to the professional exercise, associated to the phenomena.

Key words: Carpal tunnel syndrome, Pain, Dentists, Professional risks.
\end{abstract}




\section{INTRODUCCIÓN}

El Síndrome de Túnel Carpiano (STC) es la patología resultante de la compresión del nervio mediano en la muñeca. El túnel es una estructura integrada por ligamentos y huesos; el nervio mediano pasa por el túnel carpiano junto con los tendones que permiten el cierre de la misma. Cuando se tensionan, los tendones se inflaman dentro del túnel comprimiendo el nervio mediano. ${ }^{1}$ Además de los movimientos repetitivos, otros factores se pueden asociar al fenómeno como artritis, diabetes, fracturas mal alineadas, cambios hormonales, cuando se presentan posturas incomodas y al realizar fuerzas de alta tensión. También este se encuentra asociado a actividades como jugar bolos, la jardinería, la costura, en los higienistas, odontólogos, ingenieros de sistemas debido a la repetición de movimientos. ${ }^{1-4}$

El reporte más antiguo de esta condición se remonta a 1854, en donde se describió fractura distal del radio. En 1947, se describieron los signos clínicos, el diagnóstico y fisiopatología de la compresión del nervio en el túnel del carpo. En 1950, se describieron maniobras de diagnóstico como la percusión del nervio mediano en la muñeca y la prueba de flexión, que se conoce en la actualidad como la prueba de Phalen. En 1980 se describió la afección con signos de dolor y parestesia del nervio mediano. ${ }^{1,5,7}$

Entre los signos y síntomas del túnel carpiano, se presenta un hormigueo doloroso tan intenso como para perturbar el sueño, el paciente puede reportar dificultad en la movilidad de los dedos e incapacidad para agarrar objetos. Adicionalmente, se presenta sensación de hinchazón principalmente en los dedos anular, índice y la almohadilla del pulgar que tiende a atrofiarse y perder fuerza., 1,68

En un estudio realizado con odontólogos e higienista dentales se determinó que los movimientos repetitivos, junto con el mal manejo del instrumental odontológico y/o instrumentos vibratorios eran uno de los factores de riesgo de sufrir alteraciones neurológicas en las manos. ${ }^{2,5,8 .}$

En 1997, la Asociación Dental Americana (ADA) reportó que el 9.2\% de odontólogos han sido diagnosticados con alguna injuria causada por algún tipo de movimiento repetitivo. ${ }^{8}$
A pesar de las diferentes alternativas terapéuticas que se describen en la mayoría de los casos, sólo la descompresión quirúrgica de los nervios resuelve de manera definitiva las manifestaciones clínicas, , 5, 8-12 por lo tanto identificar los factores de riesgos e implementar estrategias preventivas podría constituirse como la mejor alternativa que deben seguir los profesionales de la salud oral y que deberían implementar las Administradoras de Riesgos Profesionales (ARP) sobre este grupo en particular. $^{13}$

En este estudio, se determinó la prevalencia del STC y sus factores asociados en odontólogos de la ciudad de Bucaramanga y su área metropolitana.

\section{MATERIALES Y MÉTODOS}

Se realizó un estudio observacional analítico de corte transversal cuya población estuvo conformada por 250 odontólogos de Bucaramanga y su área metropolitana, registrados en la base de datos de PUBLICAR S.A.

El tamaño de la muestra se calculó mediante la rutina simple size del paquete estadístico Epi info $6.04^{14}$ considerando las siguientes especificaciones de diseño para un estudio analítico de corte transversal: nivel de confianza del $95 \%$, poder $80 \%$, con una relación expuestos, y no expuestos $1: 1$, con una frecuencia del túnel carpiano en el grupo no expuesto del 1\%, y en expuestos del 14\%, ${ }^{2}$ una R.R. de 14 y OR de 16 por lo cual una muestra de 152 odontólogos permitirían detectar diferencias estadísticamente significativas. La muestra fue seleccionada mediante un muestreo probabilístico de tipo aleatorio simple.

Se incluyeron en el estudio odontólogos generales, y especialistas como cirujanos orales y maxilofaciales, periodoncistas, endodoncistas, odontopediatras, rehabilitadores orales, ortodoncistas y profesionales con un mínimo de un año de ejercicio profesional. Se consideraron como criterios de exclusión haber sido tratado previamente para esta patología, registrar condiciones como artritis u osteoporosis, antecedentes de fractura de mano y/o muñeca y mujeres embarazadas al momento del examen. 
En esta investigación se aplicó un instrumento tipo encuesta autodiligenciada, donde se registraron variables sociodemográficas, edad, género, especialidad, tiempo de ejercicio profesional, padecimiento de alguna enfermedad, práctica de algún deporte, uso del computador y tipo de instrumental utilizado, que se consideraron como variables riesgo; adicionalmente, se recolectaron variables relacionadas con antecedentes médicos como intensidad de dolor diurno, nocturno, entumecimiento, debilidad y dificultad para agarrar objetos, para así poder establecer ausencia o presencia del evento de interés y sus posibles factores asociados y finalmente, el diagnóstico de STC que se reportaba en otro instrumento.

Previa a la recolección de los datos se efectúo una prueba piloto con 12 odontólogos docentes de la Universidad Santo Tomás; a partir de ésta se modificaron los instrumentos, se estandarizaron los procedimientos y se definieron los tiempos para el desarrollo de las pruebas y el diligenciamiento de la encuesta.

Inicialmente, se procedió a la selección, ubicación, coordinación de la aplicación de la encuesta de autodiligenciamiento que se entregaba a cada odontólogo y a obtener el respectivo consentimiento informado. Posteriormente y para definir el diagnóstico de STC una fisioterapeuta especializada efectuó la prueba de Phalen, ${ }^{6}$ que consiste en que la persona junta sus manos palma con palma realizando una ligera presión durante un minuto con los brazos de forma vertical y el antebrazo de forma horizontal. Si el odontólogo reportaba dolor, adormecimiento 0 entumecimiento en la mano durante el minuto o menos, la prueba era positiva. Adicionalmente, la especialista realizaba movimientos en los dedos de la mano, muñeca y en la almohadilla del pulgar para establecer la presencia del síndrome en la población evaluada.

Los datos se procesaron en Excel ${ }^{15}$ por duplicado, se exportaron al paquete estadístico EPI-INFO $6.04^{14} \mathrm{y}$ mediante la rutina Validate se verificó la calidad de la digitación, finalmente se exportó al paquete STATA $8.0^{16}$ para su correspondiente análisis. El plan de análisis incluyó análisis univariado describiendo las variables mediante medidas de tendencia central y dispersión según su naturaleza y el bivariado en el que se analizó la relación de presencia de Síndrome de Túnel Carpiano (STC) con cada variable analizada mediante test de $\mathrm{chi}^{2} \mathrm{y}$ test exacto de Fischer. ${ }^{17,18}$
Para el análisis multivariado se aplicó un análisis de regresión binomial que estimó los RR $>$ (Riesgos Relativos: Razón de prevalencia) y sus respectivos intervalos de confianza del 95\%. ${ }^{19.20}$ Se definió como variable de salida STC y como variables explicatorias exclusivamente a aquellas relacionadas con factores de riesgo. Se analizó por separado cada una de estas variables en el análisis de regresión binomial bivariado inicial. A partir de este se seleccionó para el modelo final las variables que obtuvieron probabilidad igual o inferior a $\mathrm{p}=0.25$ y se forzaron en el modelo aquellas que se consideraron biológicamente significativas. ${ }^{21}$ Todo el análisis se realizó considerando un nivel de significancia de $\operatorname{Alfa}(\alpha)=0.05$

De acuerdo con la resolución 008430 de 199322 emitida por el Ministerio de Salud en el cual se establecen las normas científicas, técnicas y administrativas para investigaciones en salud y que en el articulo 11 , literal b, esta investigación se considera de riesgo mínimo, ya que se recolectaron datos a través de procedimientos comunes, consistentes en examen físico y una encuesta de auto diligenciamiento.

\section{RESULTADOS}

Se evaluaron en total 152 odontólogos con una edad promedio de $36.7 \pm 7.8$ años; el 52,6\% (80) eran hombres; el $48,7 \%$ de los odontólogos eran generales, seguido por especialistas en rehabilitación $(15,1 \%)$ y una prevalencia de STC de 15,8 \% (24) (Tabla 1).

El 54,6\% de los sujetos evaluados registraron un tiempo de ejercicio profesional $\leq 12$ años y el $98 \%$ (149) no reportaron enfermedades sistémicas. El 49,3\% (75) de las personas evaluadas no hacia ningún deporte, el 81,6\% (124) usan el computador en promedio de $152 \pm 121$ horas. El $82,9 \%$ (126) utilizan instrumental rotatorio con un tiempo superior a $120 \mathrm{~min} /$ día y el 55,3\% (84) reportan tiempos iguales o inferiores a $240 \mathrm{~min} /$ día de uso de instrumental de mano. Ninguna de las variables anteriores y relacionadas como factores de riesgo, mostró diferencias significativas a excepción del tiempo de uso del instrumental manual, pues se observó que mientras el $25 \%$ de los profesionales que registraron STC usan el instrumental de mano por un tiempo $\leq 240$ minutos, el 4,4\% (3) de los que registraron STC lo utilizan en tiempo superior a $240 \mathrm{~min} /$ día $(\mathrm{p}=0.001)$ (Tabla 2). 
Tabla 1. Descripción de la frecuencia de STC según las variables sociodemográficas.

\begin{tabular}{|c|c|c|c|c|}
\hline \multirow{2}{*}{ VARIABLE } & GLOBAL. & \multicolumn{2}{|c|}{ TÚNEL CARPIANO } & \multirow{2}{*}{$\mathbf{P}$} \\
\hline & $n=152$ & SI $(n=24)$ & No $(n=128)$ & \\
\hline \multicolumn{5}{|l|}{ EDAD (años) } \\
\hline$\leq 36$ & 82 (54\%) & $12(14.696)$ & $70(85.4 \%)$ & $0,672^{*}$ \\
\hline$>36$ & \multirow[t]{2}{*}{$70(46 \%)$} & $12(17,496)$ & $58(82,9 \%)$ & \\
\hline \multicolumn{4}{|l|}{ GÉNERO } & \\
\hline Mujeres & $72(47,4 \%)$ & $12(16,736)$ & $60(83,3 \%)$ & \multirow[t]{2}{*}{$0,778^{*}$} \\
\hline Hombres & $80(52,6 \%)$ & 12 (15\%) & $68(85 \%)$ & \\
\hline \multicolumn{5}{|l|}{ ESPECIALIDAD } \\
\hline Odontólogo general & $74(48.7 \%)$ & $10(13,5 \%)$ & $64(86,5 \%)$ & \multirow[t]{7}{*}{$0,134^{*+}$} \\
\hline Endodoncistas & $10(6,9 \%)$ & $4(40 \%)$ & $6(60 \%)$ & \\
\hline Cirujanos & 9 (5.9\%) & 1 (11.1\%) & $8(83.5 \%)$ & \\
\hline Periodoncistas & $8(5.3 \%)$ & $2(25 \%)$ & $6(75 \%)$ & \\
\hline Rehabilitadores & $23(15,1 \%)$ & $1(4.4 \%)$ & $22(95,6 \%)$ & \\
\hline Ortodoncistas & $21(13,8 \%)$ & $4(19.1 \%)$ & $17(80,9 \%)$ & \\
\hline Odontopediatras & $7(4,6 \%)$ & $2(28,6 \%)$ & $5(71,496)$ & \\
\hline
\end{tabular}

El 32,2\% (49) de los odontólogos encuestados reportaron algún nivel de intensidad de dolor diurno y el $23 \%$ de ellos (35) refirieron un dolor leve con un promedio de duración de episodio de 13,3 \pm 30,63 min/día. El 40,8\% (20) de las personas que relataron dolor diurno presentaban STC y el $61,5 \%$ (8) de los que reportaron dolor moderado presentaban STC; el 46,7\% (7) de las personas con una duración del dolor entre 46'-180'/día registraban STC. Las variables dolor diurno, intensidad y duración del episodio reportado por los odontólogos mostraron diferencias estadísticamente significativas entre los que presentaban y no presentaban STC, $\mathrm{p}<0.001$.

El 13,8\% (20) de los odontólogos encuestados reportaron algún nivel de intensidad de dolor nocturno, el 8,6\% (13) calificaron este dolor como leve, el 60\% (12/20) de las personas con dolor nocturno presentaron STC y el 66,7\% (4) de los que manifestaron dolor moderado registraron esta misma condición. La intensidad del dolor nocturno como síntoma reportado por los encuestados registró diferencia estadísticamente significativa entre los que tenían y los que no tenían STC (Tabla 3).

Al analizar otros síntomas asociados a STC se observó que el 17,1\% (26) de la población reportaba algún nivel de intensidad de debilidad, siendo esta sensación leve en el $13,8 \%$ (21). El 85,5\% (130) refirieron cierto entumecimiento de las manos, de los cuales el 68,4\% (104) reportaron un entumecimiento leve. El 9,9\% (15) manifestaron de 2 a 4 episodios nocturnos de entumecimiento en sus manos y el 13,8.\% (21) algún nivel de dificultad al agarrar objetos. Al analizar otros síntomas y su relación con la patología en estudio, se observó que el 53.8\% (14/26) de los que reportaban tener alguna nivel de debilidad en sus manos tenían STC, mientras que el 7.9\% (10/126) de los que no reportaban debilidad registraban esta condición.

El 100\% de los que reportaron debilidad moderada y severa (4 y 1, respectivamente) registraban STC, mientras que sólo el $42.9 \%$ de los que reportaban debilidad leve tenia esta condición. El 17,7\% (23/130) de los que refirieron entumecimiento en su mano presentaban STC, mientras que sólo el 4,5\% (1) de los que no reportaban entumecimiento presentaron STC. El 83,3\% (5) de la población con entumecimiento severo tenían STC, mientras que el 16,7\% (1) que reportaba este entumecimiento severo no presentaba síndrome del túnel.

Intensidad de debilidad, entumecimiento y episodios de entumecimiento nocturno mostraron diferencia estadísticamente significativa con $\mathrm{p}<0.001$. Mientras que el $47,6 \%$ (10/21), de las personas que relataban dificultad para agarrar objetos tenían STC. El 10,7\% (14) de las personas que no reportaban esta dificultad también presentaban STC con $\mathrm{p}<0.001$. El 37\% (10/27) de las personas que dejaban caer objetos tenia STC y el 11,2\% (14) que no dejaban caer objetos presentaban STC, $\mathrm{p}<0.005$ (Tabla 4). 
Tabla 2. Descripción de la frecuencia de STC según factores de riesga.

\begin{tabular}{|c|c|c|c|c|}
\hline \multirow[t]{2}{*}{ VARIABLE } & GLOBAL & \multicolumn{2}{|c|}{ TÚNEL CARPIANO } & \multirow{2}{*}{$\mathbf{P}$} \\
\hline & $N=152$ & SI $(\mathbf{n}=\mathbf{2 4})$ & NO $(\mathbf{n}=128)$ & \\
\hline \multicolumn{5}{|l|}{ TIEMPO DE EJERCICIO } \\
\hline \multicolumn{5}{|l|}{ PROFESIONAI. (años) } \\
\hline$\leq 12$ & $83(54,6 \%)$ & $13(15,7 \%)$ & $70(84,3 \%)$ & \multirow[t]{2}{*}{$0,962^{*}$} \\
\hline$>12$ & $69(45,3 \%)$ & $11(15,9 \%)$ & $58(84,1 \%)$ & \\
\hline \multicolumn{5}{|l|}{ CONDICIÓN SISTÉMICA } \\
\hline \multicolumn{5}{|l|}{ COMPROMETIDA } \\
\hline SI & $3(2 \% 6)$ & $1(33.3 \%)$ & $2(66.7 \%)$ & \multirow[t]{2}{*}{$0,405^{* *}$} \\
\hline NO & $149(98 \%)$ & $23(15.4 \%)$ & $126(84.6 \%)$ & \\
\hline \multicolumn{5}{|l|}{ DEPORTES } \\
\hline Ninguno & $75(49.3 \%)$ & $10(13,3 \%)$ & $65(86.7 \%)$ & \multirow[t]{6}{*}{$0,619^{* *}$} \\
\hline Tenis & $18(11.8 \%)$ & $2(11.1 \%)$ & $16(88,9 \%)$ & \\
\hline Baloncesto & $16(10,5 \%)$ & $2(12,5 \%)$ & $14(87,5 \%)$ & \\
\hline Bolos & $2(1,3 \%)$ & 0 & $2(100 \%)$ & \\
\hline Volleyball & $15(9,9 \%)$ & 3 (20\%) & $12(80 \%)$ & \\
\hline Otros & $26(17.1 \%)$ & $7(36,9 \%)$ & $19(73,1 \%)$ & \\
\hline \multicolumn{5}{|c|}{ USO DEL COMPUTADOR } \\
\hline SI & $124(81.6 \%)$ & $20(16.1 \%)$ & $104(83.9 \%)$ & \multirow[t]{2}{*}{$1,0^{* 4}$} \\
\hline NO & $28(18,4 \%)$ & $4(14.3 \%)$ & $24(85,7 \%)$ & \\
\hline \multicolumn{5}{|c|}{$\begin{array}{l}\text { TIEMPO DE USO DEI. } \\
\text { COMPUTADOR (Min/dia) }\end{array}$} \\
\hline$\leq 120$ & $81(53,3 \%)$ & $11(13,6 \%)$ & $70(86.4 \%)$ & \multirow{2}{*}{$0,425^{*}$} \\
\hline$>120$ & $71(46.7 \%)$ & $13(18,3 \%)$ & $58(81,7 \%)$ & \\
\hline \multirow{2}{*}{\multicolumn{5}{|c|}{$\begin{array}{l}\text { TIEMPO DE USO DEI. } \\
\text { INSTRUMENTAI. ROTATORIO } \\
\text { (Min/dia) }\end{array}$}} \\
\hline & & & & \\
\hline$\leq 120$ & $26(17.1 \%)$ & $3(11.5 \%)$ & 23 (88.5\%) & \multirow[t]{2}{*}{$0,768^{* *}$} \\
\hline$>120$ & $126(82.9 \%)$ & $21(16.7 \%)$ & $105(83.3 \%)$ & \\
\hline \multirow{2}{*}{\multicolumn{5}{|c|}{$\begin{array}{l}\text { TIEMPO DE USO DEL } \\
\text { INSTRUMENTAL MANUAL } \\
\text { (Min/dia) }\end{array}$}} \\
\hline & & & & \\
\hline$\leq 240$ & $84(55.3 \%)$ & 21 (25\%) & $63(75 \%)$ & \multirow[t]{2}{*}{$0,001^{* *}$} \\
\hline$>240$ & $68(44,7 \%)$ & $3(4.4 \%)$ & $65(95.6 \%)$ & \\
\hline
\end{tabular}

El modelo de regresión binomial no ajustado mostró que el compromiso sistémico presentaba una asociación 2.15 IC 95\% [0.41-11.17] mayor de tener STC entre los que no tenían compromiso sistémico sin que se registraron diferencias estadísticamente significativas $(\mathrm{p}=0.359)$. Además, se observó una relación de 0.17 menor de tener STC entre los que reportaban un uso del instrumental manual de $>240$ minutos IC [0.054-0.56] siendo estadísticamente significativa $(\mathrm{p}=0.004)$ (Tabla 5$)$.

El modelo final ajustado permite asociar al STC con compromiso sistémico, con R.R. 6,38 IC [1.91-21.26] $\mathrm{p}=0.003$ en las personas con compromiso sistémico que entre las que no lo presentaban. También se relacionó con el uso de instrumental rotatorio por tiempo mayor a $120 \mathrm{minu}-$ tos mostró un R.R. de 1.81 IC 95\% [0.59-5.49]; sin embargo, no se evidenciaron diferencias estadísticamente significativas $(p=0,292)$. Finalmente, se asoció en forma negativa con el uso de instrumental manual por tiempo mayor a 240 minutos con un R.R. de 0.13 IC 95\% [0.04-0.448] observándose como una variable estadísticamente significativa $\mathrm{p}=0.001$ (Tabla 6). 
Tabla 3. Descripción del dolor asociada aSTC.

\begin{tabular}{|c|c|c|c|c|}
\hline \multirow{2}{*}{ VARIABLE } & GLOBAL & \multicolumn{2}{|c|}{ TÚNEI. CARPLANO } & \multirow{2}{*}{$\mathbf{P}$} \\
\hline & $\mathbf{n}=152 \quad \mathbf{n}(\%)$ & SI $(\mathbf{n}=24)$ & NO $(n=128)$ & \\
\hline \multicolumn{5}{|c|}{ INTENSIDAD DEL. DOLOR DIURNO } \\
\hline Ninguno & $103(67.8 \%)$ & $4(3.9 \%)$ & $99(96.1 \%)$ & $<0,001^{* *}$ \\
\hline Leve & $35(23 \%)$ & $11(31.4 \%)$ & $24(68.6 \%)$ & \\
\hline Moderado & $13(8.6 \%)$ & $8(61.5 \%)$ & $5(38.5 \%)$ & \\
\hline Severo & $1(0.6 \%)$ & $1(100 \%)$ & 0 & \\
\hline \multicolumn{5}{|c|}{ DURACIÓN DEL. EPISODIo (minutos) } \\
\hline $0 \cdot 2 g^{\prime}$ & $121(79.696)$ & $10(8.3 \%)$ & $111(91.7 \%)$ & $<0,001^{*}$ \\
\hline $30^{\prime}-45^{\prime}$ & $16(10,5 \%)$ & $7(43,8 \%)$ & $9(56,2 \%)$ & \\
\hline $46^{\prime}-180^{\prime}$ & $15(9,9 \%)$ & $7(46,7 \%)$ & $8(53.3 \%)$ & \\
\hline \multicolumn{5}{|c|}{ INTENSIDAD DEL. DOLOR NOCTURNO } \\
\hline Ninguno & $132(86.806)$ & $12(9.1 \%)$ & $120(90.9)$ & $<0,001^{\circ}$ \\
\hline Leve & $13(8.6 \%)$ & $7(53.8 \%)$ & $6(46.2 \%)$ & \\
\hline Moderado & $6(4 \%)$ & $4(66.7 \%)$ & $2(33.3 \%)$ & \\
\hline Severo & $1(0.6 \%)$ & $1(100)$ & 0 & \\
\hline
\end{tabular}

Tabla 4. Descripción de otros sintomas asociados a STC.

\begin{tabular}{|c|c|c|c|c|}
\hline \multirow{2}{*}{ VARIABLE } & GLOBAL. & \multicolumn{2}{|c|}{ TÚNEL CARPIANO } & \multirow{2}{*}{$\mathbf{P}$} \\
\hline & $n=152 \quad n(\%)$ & SI $(\mathbf{n}=\mathbf{2 4})$ & NO $(n=128)$ & \\
\hline \multicolumn{5}{|c|}{ INTENSIDAD DE LA DEBILIDAD } \\
\hline Ninguno & $126(82.9 \%)$ & $10(7,9 \%)$ & $116(92.1 \%)$ & $<0,001^{* *}$ \\
\hline Leve & $21(13,8 \%)$ & $9(42,9 \%)$ & $12(57,1 \%)$ & \\
\hline Moderado & $4(2,6 \%)$ & $4(100 \%)$ & 0 & \\
\hline Severo & $1(0.6 \%)$ & $1(100 \%)$ & 0 & \\
\hline \multicolumn{5}{|c|}{ INTENSIDAD DEL ENTUMECIMIENTO } \\
\hline Ninguno & $22(14,5 \%)$ & $1(4.5 \%)$ & $21(95.5 \%)$ & $<0,001^{* *}$ \\
\hline Leve & $104(68.4 \%)$ & $10(9.6 \%)$ & $94(90.4)$ & \\
\hline Moderado & $20(13,2 \%)$ & $8(40 \%)$ & $12(60 \%)$ & \\
\hline Severo & $6(4 \%)$ & $5(83.3 \%)$ & $1(16.796)$ & \\
\hline \multicolumn{5}{|c|}{ EPISODIO DE PERCEPCIÓN DEL. ENTUMECIMIENTO NOCTURNO } \\
\hline $0-1$ & $136(89.5 \%)$ & $14(10.3 \%)$ & $122(89.7 \%)$ & $<0,001^{* 4}$ \\
\hline $2 \cdot 4$ & $15(9.9 \%)$ & $9(60 \%)$ & $6(30 \%)$ & \\
\hline $5-6$ & $1(0.6 \%)$ & $1(100 \%)$ & 0 & \\
\hline \multicolumn{5}{|c|}{ INTENSIDAD DE DIFICULTAD PARA AGARRAR OBJETOS } \\
\hline Ninguno & $131(86.2 \%)$ & $14(10.7 \%)$ & $117(89.3 \%)$ & $<0,001^{* *}$ \\
\hline Leve & 13 (8.6\%) & $9(69.2 \%)$ & $4(30.896)$ & \\
\hline Moderado & $8(5.2 \%)$ & $1(12.5 \%)$ & $78(7.5 \%)$ & \\
\hline \multicolumn{5}{|c|}{ FRECUENCIA DE CAIDA DE OBJETOS } \\
\hline Nunca & $125(82.2 \%)$ & $14(11,2 \%)$ & $111(88.8 \%)$ & $0,005^{* *}$ \\
\hline Poco & $22(14,6 \%)$ & $8(36.4 \%)$ & $14(63.6 \%)$ & \\
\hline Regular & $5(3,3 \%)$ & $2(40 \%)$ & $3(60 \%)$ & \\
\hline
\end{tabular}


Tabla 5. Modelo de regresión binomial de STC y de los factores de riesgo asociado. Anúlisis bivariado.

\begin{tabular}{lccc}
\hline \multicolumn{1}{c}{ VARIABLE } & R.R. & IC 95\% & P \\
\hline Edad 236 & 1.17 & $0.56-2.44$ & 0.673 \\
Mujeres & 1.11 & $0.53-2.31$ & 0.778 \\
Odontólogo especialista & 1.32 & $0.62-2.80$ & 0.456 \\
>12 años de ejercicio profesional & 1.01 & $0.48-2.12$ & 0.962 \\
Compromiso sistémico & 2.15 & $0.41-11.17$ & 0.359 \\
Volleyball & 1.5 & $0.47-4.8$ & 0.495 \\
Uso del computador & 1.12 & $0.42-3.04$ & 0.811 \\
Tiempo de uso del computador & 1.34 & $0.64-2.81$ & 0.427 \\
mayor de 120 minjdia & & & \\
Tiempo de uso del instrumental & 1.44 & $0.46-4.48$ & 0.525 \\
rotatorio mayor de 240 min/Dia & & & \\
Tiempo de uso del instrumental & 0.17 & $0.054-0.56$ & 0.004 \\
manual mayor de 240 min/Dia & & & \\
\end{tabular}

Tabla 6. Modelo de regresión binomial final ajustado por los factores de riesgo asociado a STC.

\begin{tabular}{lccc}
\hline \multicolumn{1}{c}{ VARIABLE } & R.R. & IC 95\% & P \\
\hline Tiempo de uso del Instrumental & 0.13 & $0.44-0.448$ & 0.005 \\
manual $>240$ min/dia & 6.38 & $1.91-21.26$ & 0.003 \\
$\begin{array}{l}\text { Compromiso sistemico } \\
\text { Tiempo de uso del instrumental }\end{array}$ & 1.81 & $0.59-5.49$ & 0.298 \\
rotatorio $>120$ min/dia & & & \\
\hline
\end{tabular}

\section{DISCUSIÓN}

En el presente estudio en odontólogos de Bucaramanga registrados en la base de datos de PUBLICAR S.A., se observó una prevalencia de STC en el 15,8\% de la población evaluada, lo que coincide con lo expresado por Horstman y colaboradores, quienes reportaron una prevalencia del $12 \%$ en odontólogos. ${ }^{2}$

En la revisión de la literatura se encontró que en un estudio realizado con odontólogos e higienistas dentales se determinó que los movimientos repetitivos, junto con el mal manejo del instrumental odontológico y/o rotatorio eran uno de los factores de riesgo de sufrir alteraciones neurológicas en las manos. ${ }^{2-5}$

Adicionalmente, se observó en esta investigación una asociación mayor de tener túnel carpiano en odontólogos que reportaban compromiso sistémico, hecho que también se hace evidente en lo reportado por Fish y MorrysAllen quienes afirmaban que enfermedades como la artritis, diabetes, retención de líquidos, gota y fractura de mano y muñeca se han asociado a la compresión del nervio mediano. ${ }^{5}$

Es importante resaltar que el uso prolongado del instrumental rotatorio se relacionó con el evento de interés, sin embargo es contradictorio observar que el uso prolongado de instrumental manual registró una relación menor de presencia de STC. Es evidente que con estudios analíticos de corte transversal no es posible establecer relaciones de causalidad de los factores de riesgo con STC y sólo relaciones de asociación podrían definirse en el presente estudio. $22-25$

Por tanto este último resultado podría estar más relacionado como un efecto del túnel carpiano y no con la causa pues es probable que las personas con STC estén utilizando instrumental manual por menos tiempo por el efecto del dolor y la molestia que genera este tipo de patologías.

El rango de edad más frecuente en el que se observó esta patología fue de 36 años y más sin presentarse diferencias significativas por género. Ramírez y colaboradores, reportaron que según género es tres veces mayor la presencia de túnel carpiano en las mujeres que en los hombres ${ }^{11}$ hecho que no es perceptible en el presente estudio; sin embargo, el evento túnel si se relaciona con la edad pues los mismos autores afirman que el diagnóstico de STC era evidente entre la tercera y cuarta década de la vida.

Andersen y colaboradores dedujeron en un estudio que el trabajo prolongado en el computador es propicio para el padecimiento del STC. ${ }^{26} \mathrm{La}$ presente investigación no encontró asociaciones para uso del computador en odontólogos.

Una de las ventajas del proyecto fue la relación estadísticamente significativa que se pudo establecer entre los síntomas reportados por los odontólogos y el diagnóstico del evento que fue realizado por una profesional especializada; lo que podría indicar que las ARP podrían implementar estrategias de tamizaje en los odontólogos, ${ }^{9}$ fundamentados en el autoreporte de síntomas relacionados con dolor, entumecimiento y debilidad a nivel de la mano así como dejar caer objetos en forma involuntaria.

Al considerar las limitaciones de este estudio se encontró que por ser un formato auto diligenciado se presentaron inconsistencias en los datos que dificultaron levemente la tabulación.

Es importante resaltar que este es el primer trabajo de investigación que se realiza en odontólogos de Bucaramanga y su área metropolitana, relacionado con el Síndrome de Túnel Carpiano, lo que conlleva a la necesidad de formular programas de promoción y prevención de auto cuidado frente al fenómeno en estudio. 


\section{BIBLIOGRAFÍA}

1. Gutierrez GC. Gargollo OC, Jiménez MY. Manejo del síndrome del tune del carpo en el hospital general "Dr. Manuel Gea González». Cir Plast 2002; 12: 25 - 30. URL disponible en: http://www.medigraph.com/ español/e-cp2002/e-cp02-1/er-cp021e.htm

2. Horstman SW, Horstman BC, Horstman FS. Ergonomic risk factors associated with the practice of dental hygiene: A preliminary study. Am Soc Safety Eng 1997; 49 - 53.

3. Alexopoulos E, Stathi IC, Charizani F. Prevalence of musculoskeletal disorders in dentist. BMC musculoskeletal disorders 2004.; 5: 1 - 8 [fecha de acceso: febrero de 2005]. URL disponible en: http:/| www.Biomedcentral.com/content/pdf/1471-2474-5-16.pdf

4. Szymanska J. Disorders of the musculoskeletal system among dentist from the aspect of ergonomics and prophylaxis. Ann Agric Environ Med 2002; 9: 169 - 173.

5. Fish DR, Morris-Allen D. Musculoskeletal disorders in dentist. NY State Dent J 1998; 64: 44 -48.

6. Prigane VW, Henry SM. The Relationship among Five Common Carpal Tunnel Syndrome Test and the Severity of Carpal Tunnel Syndrome. J. Hand Ther 2003; 16: 225 - 236.

7. D'arcy CA, McGee S. The rational clinical examination. Does this patient have carpal tunnel syndrome? JAMA 2000; 283: 3110 - 3117.

8. Ligh, RQ. Preventing cumulative trauma injury carpal tunnel syndrome. J Calif Dent Assoc. 2002; 30: 671, 674 - 678.

9. Bland JDP, Rudolfer SM. Clinical surveillance of carpal tunnel syndrome in two areas of the United Kingdom, 1991-2001. J Neurol Neurosurg Psychiatry 2003; 74: 1674 - 1679.

10. Gerritsen AA, Korhals-de Bos IB, Laboryie PM, de Vet HC, Scholten RJ, Bouter LM. Splinting for carpal tunnel syndrome: prognostic indicators of success. J Neurol Neurosurg Psychiatry 2003; 74: 1342 - 1344.

11. Ramírez EA, Cuenca-Pardo J, Martínez M, Chávez J, Corral MA. Manejo del túnel del carpo a través de incisiones mínimas y cuchillo luminoso, en el Hospital de Ortopedia "Dr. Victorio de la Fuente Narváez" de IMSS. Cir Plast 2002; 12: 100 - 103. URL disponible en: http:// www.medigraphic.com/pdfs/cplast/cp-2002/cp023c.pdf

12. Atroshi I, Gummesson C, Johnsson R, McCabe SJ Ornstein E. Severe carpal syndrome potentially needing surgical treatment in a general population. J Hand Surg; 2003: 28: 639 - 644.

13. Burke FD, Ellis J, McKenna, Bradley MJ. Primary care management of carpal tunnel syndrome. Postgrad Med J 2003; 79: 433 - 437.

14. CDC-OMS. Epi-Inf 6 Versión 6.04a Nov 1996.

15. Microsoft Excel 5.0, Microsoft Corporation 1997.

16. Stata corp 2003. Stata statistical software. Release 8.0 College station, T.X: Stata Corporation.
17. Pagano M, Gauvreau K, Principles of Biostatistics. Duxbury Press. Belmont p. 35 - 44, 257- 263.

18. Norman GR, Streiner DL. Bioestadística. Mosby/Doyma Libros. Madrid. 1996 p 129 - 147.

19. Wacholder S. Binomial Regression in GLIM: estimating risk and risk differences. Am J Epidemiol 1986; 123: 174 - 184.

20. Zochetti C, Consonni D, Vertáis PA. Relationship between prevalence rate ratios and odds ratios in cross-sectional studies. Int J Epidemiol 1997; 26: 220 - 223.

21. Bautista L. Métodos avanzados de análisis. Bucaramanga 2002.

22. República de Colombia. Ministerio de Salud. Resolución No. 008430 del 4 de octubre de 1993.

23. Kelsey, JL. Douglas TH. Methods in observational epidemiology. N.Y. Oxford University Press, 1986 Cap. 10 (Cross-Sectional another types of studies) p. 244- 257.

24. Feinstein, AR. Clinical biostatistics. The architecture of crosssectional research (part I). Clin Pharmacol Ther 1978; 23: 81 - 96.

25. Feinstein, AR. Clinical biostatistics. The architecture of crosssectional research (Conclusion). Clin Pharmacol Ther 1978: 481 - 493.

26. Andersen JH, Thomsen JF, Overgaard E, Lassen CF, Brandt LPA, Vilstrup I, Kryger AI, Mikkelsen S. Computer use and carpal tunnel syndrome: a 1-year follow up study. JAMA 2003; 289: 2963 - 2969.

80

Ustasalud Odontología 2005; 4: 73 - 80 Rodríguez A. y col. 